\title{
Nonlinear Response of High Arch Dams to Nonuniform Seismic Excitation Considering Joint Effects
}

\author{
Masoomeh Akbari, ${ }^{1}$ Mohammad Amin Hariri-Ardebili, ${ }^{2}$ and Hasan Mirzabozorg ${ }^{1}$ \\ ${ }^{1}$ Department of Civil Engineering, K. N. Toosi University of Technology, P.O. Box 15875-4416, Tehran, Iran \\ ${ }^{2}$ Department of Civil Environmental and Architectural Engineering, University of Colorado at Boulder, \\ P.O. Box 80309-0428, Boulder, CO, USA
}

Correspondence should be addressed to Hasan Mirzabozorg; mirzabozorg@kntu.ac.ir

Received 1 September 2012; Accepted 18 November 2012

Academic Editor: Khoa Le

Copyright (c) 2013 Masoomeh Akbari et al. This is an open access article distributed under the Creative Commons Attribution License, which permits unrestricted use, distribution, and reproduction in any medium, provided the original work is properly cited.

Nonuniform excitation due to spatially varying ground motions on nonlinear responses of concrete arch dams is investigated. A high arch dam was selected as numerical example, reservoir was modelled as incompressible material, foundation was assumed as mass-less medium, and all contraction and peripheral joints were modelled considering the ability of opening/closing. This study used Monte-Carlo simulation approach for generating spatially nonuniform ground motion. In this approach, random seismic characteristics due to incoherence and wave passage effects were investigated and finally their effects on structural response were compared with uniform excitation at design base level earthquake. Based on the results, nonuniform input leads to some differences than uniform input. Moreover using nonuniform excitation increase, stresses on dam body.

\section{Introduction}

Seismic analysis of long span structures has been done with uniform excitation, whereas the motion of the ground changes, in both amplitude and frequency, as the earthquake waves travel with a finite speed away from their source. Variations in the ground motion arise mainly from three sources, for example, the wave passage effect, the incoherence effect, and the site-response effect [1]. In this paper wave passage and incoherence effects were considered as nonuniform input sources. Many researchers have investigated the response of long span structures to asynchronous support excitations. Effect of asynchronous and multiple supports inputs in analysis of beams and bridges have been considered by Harichandran et al. [2]. They studied the effect of spatial variability of ground motion on existing long span suspension bridges. Allam and Datta [3] presented a nonstationary random vibration response evaluation of a cable-stayed bridge model subjected to spatially variable excitations. Zhang et al. [4] investigated a random vibration algorithm for the seismic response analysis which accurately accounts for the spatial variability effects of wave passage, incoherence, and site response. Although a lot of works have been done in seismic analysis of concrete arch dams, a limited of them has been investigated effects of asynchronous input on the seismic behaviour of dams. Bayraktar et al. $[5,6]$ was analysed the effect of wave propagation on the response of Sariyar concrete gravity dam. They reported that horizontal, vertical, and shear stresses in the foundation generally increased with the decreasing of propagation velocity. Maeso et al. [7] investigated behaviour of an arch dam subjected to incident body ( $\mathrm{P}_{-}, \mathrm{SV}_{-}$, and $\mathrm{SH}_{-}$) and surface (Rayleigh) waves. Alves and Hall $[8,9]$ analyzed the effect of spatially variable excitations on the nonlinear response of Pacoima dam using seismic data. Results showed that uniform excitations stresses and joint opening were largest in the centre part of the dam away from the abutments. The stresses along the abutments and in the centre of the downstream face of the dam were dominated by nonuniform excitation. 
In present study Monte-Carlo simulation approach was used for generating spatially nonuniform ground motion for nonlinear analysis of a high concrete arch dam. DEZ dam was selected as numerical example to investigate wave travelling and incoherence effects in dam-reservoir-foundation system. Reservoir was modelled as incompressible material, and foundation was supposed as mass-less medium. In addition all contraction and peripheral joints were modelled based on as-built drawings.

\section{Generation of Spatially Variation Earthquake Ground Motions (SVEGM)}

2.1. m-Variant Nonstationary Stochastic Ground Motion Process. The spectral-representation methodology is given considering the $1 \mathrm{D}-\mathrm{mV}$ (one-dimensional, $m$-variant) nonstationary ground motion stochastic vector process [10]. To be specific, consider an $n$-variant non-stationary stochastic vector process with component $f_{j}^{0}(t)(j=1,2, \ldots, m)$ having mean value equal to zero; that is, $\varepsilon\left[f_{j}^{0}(t)\right]=0(j=1,2, \ldots$, $m$ ) and cross-spectral density matrix given by

$$
S^{0}(\omega, t)=\left[\begin{array}{cccc}
S_{11}^{0}(\omega, t) & S_{12}^{0}(\omega, t) & \cdots & S_{1 m}^{0}(\omega, t) \\
S_{21}^{0}(\omega, t) & S_{22}^{0}(\omega, t) & \cdots & S_{2 m}^{0}(\omega, t) \\
\vdots & \vdots & \ddots & \vdots \\
S_{m 1}^{0}(\omega, t) & S_{m 2}^{0}(\omega, t) & \cdots & S_{m m}^{0}(\omega, t)
\end{array}\right]_{m \times m}
$$

Specifically for the case of earthquake ground motion, the elements of the cross-spectral density matrix with evolutionary power can be expressed in the following special form:

$$
\begin{array}{r}
S_{j j}^{0}(\omega, t)=\left|A_{j}(\omega, t)\right|^{2} S_{j}(\omega), \quad(j=1,2, \ldots, m), \\
S_{j j}^{0}(\omega, t)=A_{j}(\omega, t) A_{k}(\omega, t) \sqrt{S_{j}(\omega) S_{k}(\omega)} \Gamma_{j k}(\omega), \\
(j, k=1,2, \ldots, m),
\end{array}
$$

where $A_{j}(\omega, t)$ and $S_{j}(\omega)(j=1,2, \ldots, m)$ are the (nonseparable) modulating function and the (stationary) power spectral density function of component $f_{j}^{0}(t)(j=1,2, \ldots, m)$, respectively, and $\Gamma_{j k}(\omega)(j, k=1,2, \ldots, m ; j \neq k)$ is the complex coherence function describing the correlation structure between the components of the stationary vector process. They are given by

$\Gamma_{j k}(\omega)=\gamma_{j k}(\omega) \exp \left[-i \frac{\omega \xi_{j k}}{v}\right], \quad(j, k=1,2, \ldots, m ; j \neq k)$,

where $\gamma_{j k}(\omega)(j, k=1,2, \ldots, m ; j \neq k)$ are the (stationary) coherence functions between $f_{j}^{0}(t)$ and $f_{k}^{0}(t) ; \exp \left[-i\left(\omega \xi_{j k} /\right.\right.$ $v)$ ] is the wave propagation term where $\xi_{j k}(\omega)$ is the distance between points $j$ and $k$, and $v$ is the velocity of wave propagation.

For the special case of uniformly modulated nonstationary stochastic vector process, the modulating functions are independent of the frequency, that is

$$
A_{j}(\omega, t)=A_{j}(t), \quad(j=1,2, \ldots, m)
$$

In such a case, the three components of the non-stationary stochastic vector process are expressed as

$$
f_{j}^{0}(t)=A_{j}(t) g_{j}^{0}(t), \quad(j=1,2, \ldots, m)
$$

where $g_{j}^{0}(t)(j=1,2, \ldots, m)$ are the components of a stationary stochastic vector process and cross-spectral density matrix given by

$$
S^{0}(\omega)=\left[\begin{array}{cccc}
S_{1}(\omega) & \sqrt{S_{1}(\omega) S_{2}(\omega)} \Gamma_{12}(\omega) & \ldots & \sqrt{S_{1}(\omega) S_{m}(\omega)} \Gamma_{1 m}(\omega) \\
\sqrt{S_{2}(\omega) S_{1}(\omega)} \Gamma_{21}(\omega) & S_{2}(\omega) & \ldots & \sqrt{S_{2}(\omega) S_{m}(\omega)} \Gamma_{2 m}(\omega) \\
\vdots & \vdots & \ddots & \vdots \\
\sqrt{S_{m}(\omega) S_{1}(\omega)} \Gamma_{m 1}(\omega) & \sqrt{S_{m}(\omega) S_{2}(\omega)} \Gamma_{m 2}(\omega) & \ldots & S_{m}(\omega)
\end{array}\right]
$$

2.2. Simulation Formula for $m$-Variant Nonstationary Stochastic Process. According to the algorithm presented by Deodatis $[11,12]$, in order to simulate the $1 \mathrm{D}-\mathrm{mV}$ non-stationary ground motion vector process, $f_{0}(t)$ the evolutionary cross-spectral density matrix $S_{f}^{0}(\omega, t)$ is first decomposed at every time instant $t$ using Cholesky's method into the following product:

$$
S_{f}^{0}(\omega, t)=H(\omega, t) H^{T *}(\omega, t)
$$

where $H(\omega, t)$ is a lower triangular matrix, and the superscript $T$ denotes the transpose of a matrix. $H(\omega, t)$ is written as

$$
H(\omega, t)=\left[\begin{array}{cccc}
H_{11}(\omega, t) & 0 & \cdots & 0 \\
H_{21}(\omega, t) & H_{22}(\omega, t) & \cdots & 0 \\
\vdots & \vdots & \ddots & \vdots \\
H_{m 1}(\omega, t) & H_{m 2}(\omega, t) & \cdots & H_{m m}(\omega, t)
\end{array}\right]
$$


Using Cholesky's decomposition method, the diagonal elements of $H(\omega)$ are real and nonnegative functions of $\omega$ while the off-diagonal elements are generally complex functions of $\omega$. The elements of $H(\omega)$ can be written in polar form as:

$$
H_{j k}(\omega)=\left|H_{j k}(\omega)\right| e^{i \theta_{j k}(\omega)}, \quad(j>k),
$$

where,

$$
\theta_{j k}(\omega)=\tan ^{-1}\left(\frac{\operatorname{Im}\left[H_{j k}(\omega)\right]}{\operatorname{Re}\left[H_{j k}(\omega)\right]}\right) .
$$

Once the matrix $S^{0}(\omega)$ is decomposed according to (7), (8), and (9), the stationary stochastic vector process $g_{j}^{0}(t)(j=$ $1,2, \ldots, m)$ can be simulated by the following series as $N \rightarrow$ $\infty$ :

$$
\begin{array}{r}
g_{j}(t)=2 \sum_{n=1}^{m} \sum_{l=1}^{N}\left|H_{j n}\left(\omega_{l}\right)\right| \sqrt{\Delta \omega} \cos \left[\omega_{l} t-\theta_{j n}\left(\omega_{l}\right)+\phi_{n l}\right], \\
(j=1,2, \ldots, m),
\end{array}
$$

where,

$$
\begin{gathered}
\omega_{l}=l \Delta \omega, \quad(l=1,2, \ldots, N), \\
\Delta \omega=\frac{\omega_{u}}{N}, \\
\theta_{j n}\left(\omega_{l}\right)=\tan ^{-1}\left(\frac{\operatorname{Im}\left[H_{j n}\left(\omega_{l}\right)\right]}{\operatorname{Re}\left[H_{j n}\left(\omega_{l}\right)\right]}\right) .
\end{gathered}
$$

The quantities $\left\{\phi_{n l}\right\}(n=1,2, \ldots, m ; l=1,2, \ldots, N)$ appearing in (11) are $n$ sequences of independent random phase angles distributed uniformly over the interval $[0,2 \pi]$. In (13), $\omega_{u}$ represents an upper cut-off frequency beyond which the elements of the cross-spectral density matrix in (1) may be assumed to be zero for anytime instant $t$. As such, $\omega_{u}$ is a fixed value and hence $\Delta \omega \rightarrow 0$ as $N \rightarrow \infty$ so that $N \Delta \omega=\omega_{u}$.

In order to generate the $i$ th sample $g_{j}^{(i)}(t)(j=1,2, \ldots, m)$ of the stationary stochastic vector process $g_{j}(t)(j=$ $1,2, \ldots, m)$ one replaces the $m$ sequences of random phase angles $\left\{\phi_{m l}\right\}(n=1,2, \ldots, m ; l=1,2, \ldots, N)$ in (11) with their respective $i$ th realizations of $\left\{\phi_{n l}\right\}(m=1,2, \ldots, m ; l=$ $1,2, \ldots, N)$ as follows:

$$
\begin{array}{r}
g_{j}^{(i)}(t)=2 \sum_{n=1}^{m} \sum_{l=1}^{N}\left|H_{j n}\left(\omega_{l}\right)\right| \sqrt{\Delta \omega} \times \cos \left[\omega_{l} t-\theta_{j n}\left(\omega_{l}\right)+\phi_{n l}^{(i)}\right] \\
(j=1,2, \ldots, m) .
\end{array}
$$

The corresponding $i$ th realization of the non-stationary vector process $f_{j}^{(i)}(t)(j=1,2, \ldots, m)$ is calculated by multiplying the $i$ th realization of the stationary process $g_{j}^{(i)}(t)(j=1,2, \ldots, m)$ by the modulating functions $A_{j}(t)(j=1,2, \ldots, m)$ as follows:

$$
f_{j}^{(i)}(t)=A_{j}(t) g_{j}^{(i)}, \quad(j=1,2, \ldots, m) .
$$

\section{Numerical Modeling of Dam-Reservoir-Foundation System}

DEZ arch dam with $203 \mathrm{~m}$ height in Iran was selected as numerical example. Crest length is $240 \mathrm{~m}$, thickness at the crest level is $4.5 \mathrm{~m}$, and its maximum thickness at the base is $21 \mathrm{~m}$. Finite-element idealization prepared for the dam, foundation rock and reservoir is shown in Figure 1, which consists of 792 solid elements for modelling dam and concrete saddle (Pulvino), 3770 solid elements for simulation of mass-less foundation rock, and 3660 fluid elements in reservoir domain [13]. It is noteworthy that all the solid elements are displacement-based eight-node elements with three degrees of translational freedom at each node. Farend boundary of the foundation is at the distance from the dam which is about 2 times the height of the dam body in all direction. All contraction and peripheral joints were simulated based on as-built drawings using $3 \mathrm{D}$ node-to-node contact elements [14]. The concrete dam is assumed to be with the following material properties: $\rho_{d}=2400 \mathrm{~kg} / \mathrm{m}^{3}, v_{d}=$ 0.2 and $E_{d}=40 \mathrm{GPa}$. The foundation material is assumed to be $v_{d}=0.2$ and same density. In addition, reservoir water density is assumed to be $1000 \mathrm{~kg} / \mathrm{m}^{3}$, sound velocity is $1440 \mathrm{~m} / \mathrm{s}$ in water, and wave reflection coefficient for reservoir around boundary is supposed 0.8 , conservatively.

\section{Loading}

Applied loads on the system are dam body self-weight, hydrostatic pressure in normal water level, and seismic load. In addition, thermal load corresponding to summer condition is applied on the dam body. It is worthy to note that thermal load applied on the structure has been extracted from calibrated thermal transient analyses conducted using real data at the dam site taking into account solar radiation on exposed surfaces of the dam body [15]. Acceleration response spectrum of design base level (DBL) for horizontal direction is shown in Figure 2. Also time history of horizontal component of generated ground motion for uniform excitation is shown in this figure. It is notable that in the case of uniform excitation exerted ground motion in all areas is the same with the first set of nonuniform excitation.

In nonuniform ground motion the system is excited at foundation boundaries using 14 sets of simulated ground motion records compatible with DBL spectrum in upstreamdownstream direction. Based on presented formulation in previous step, a computer program was developed for generating nonuniform ground motions. The program is capable of producing different nonuniform acceleration time histories considering wave-passage and incoherence effects according to target spectrum and solving the general equation of differential support motion. The first effect is raises by the limited wave propagation velocity, depending on their relative distances away from the source. The second effect is due to reflections and refractions of seismic waves through the soil during their propagation that leads to change in amplitude and frequency away from their source. $\beta$ Newmark method is utilized to solve the coupled nonlinear 


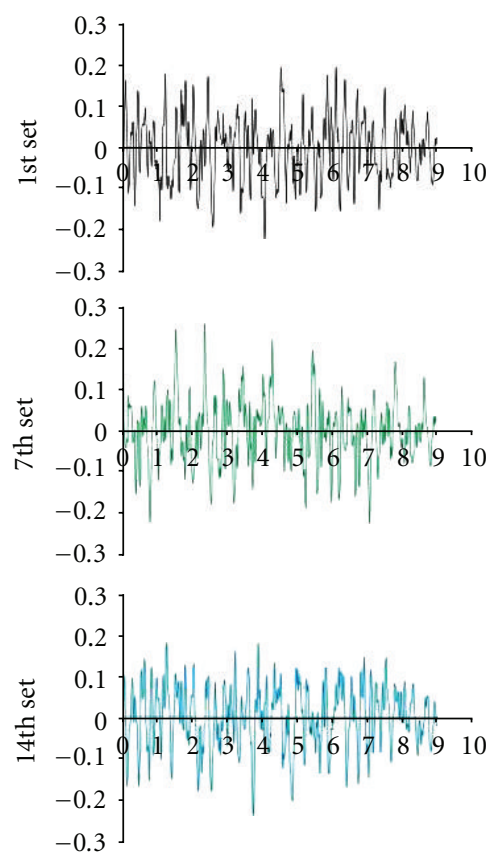

(a)

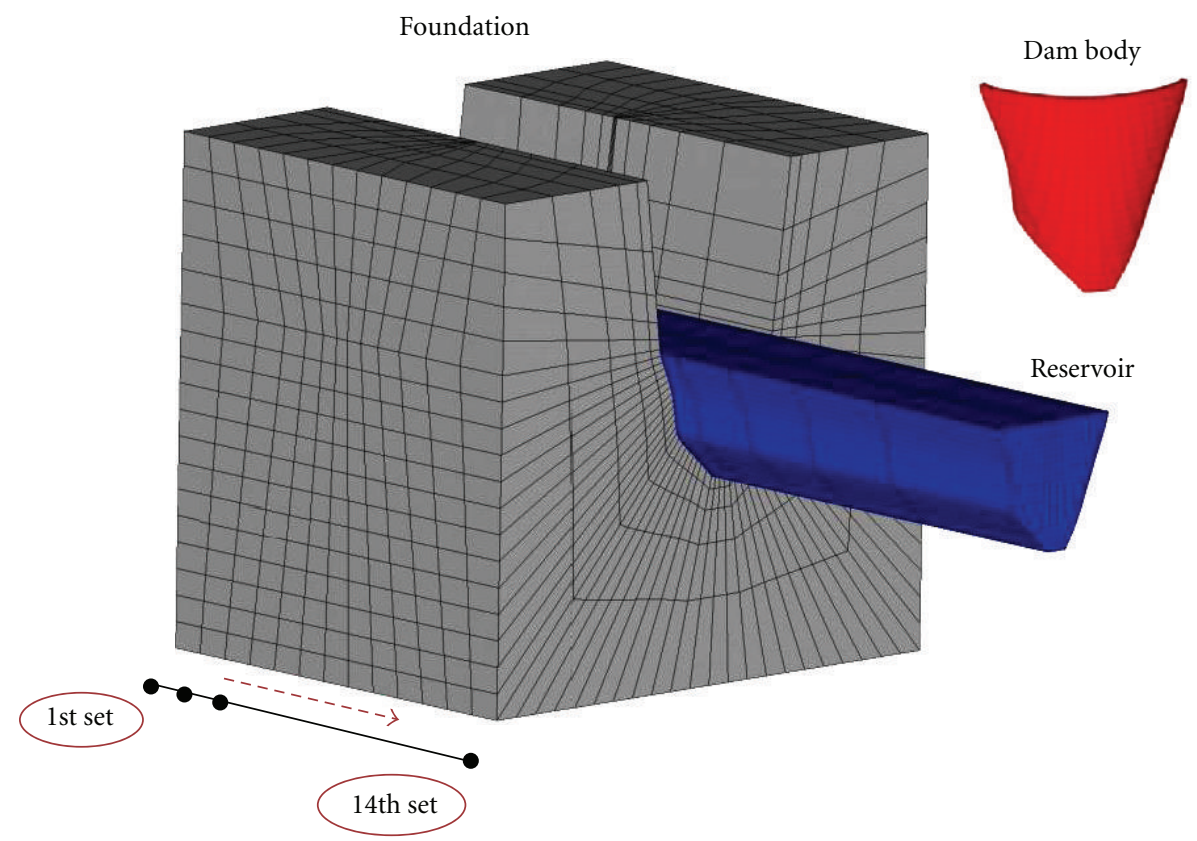

(b)

FIGURE 1: Finite element model of coupled system and time history of nonuniform ground motions at various locations of foundation.

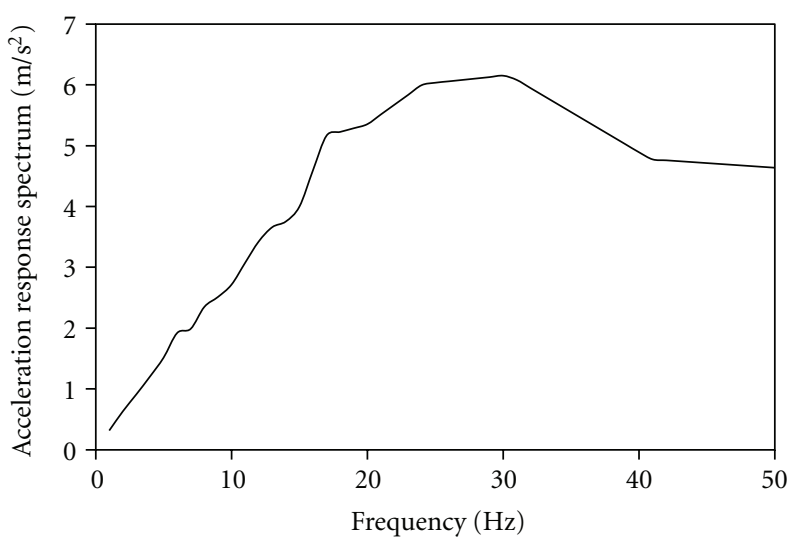

(a)

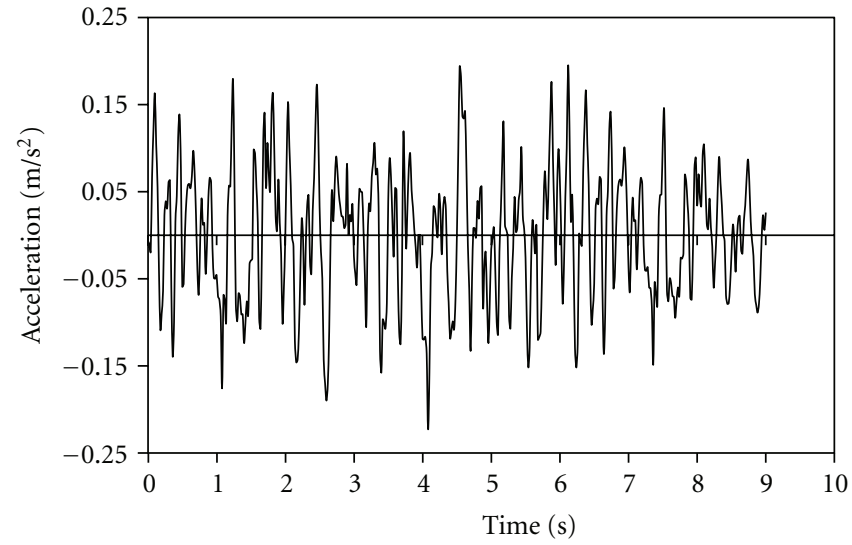

(b)

FIGURE 2: (a) Acceleration response spectrum for horizontal component of DBL, (b) Horizontal component of acceleration time history in uniform excitation.

problem of dam-reservoir-foundation model. The structural damping about $5-10 \%$ is usually used in order to dynamic analysis of concrete dams based on the level of excitation and also level of nonlinearity of the system (especially mass concrete). Considering that in the current case the coupled system is analysed in low excitation level (DBL) and also the material properties of mass concrete are assumed to be linear elastic (still there is joint nonlinearity in terms of opening and sliding), it was decided to choose structural damping as $5 \%$ of critical damping.

\section{Results and Discussion}

In this section results of displacement obtained from uniform and nonuniform analyses are compared with each other at crest point of crown cantilever. Based on Figure 3, although general trend of displacement time history is similar in both cases, in nonuniform excitation maximum displacement is happened at $t=2.9 \mathrm{~s}$, and in uniform excitation the maximum value is belonging to $t=5.1 \mathrm{~s}$. In addition uniform excitation leads to higher value than nonuniform input. 


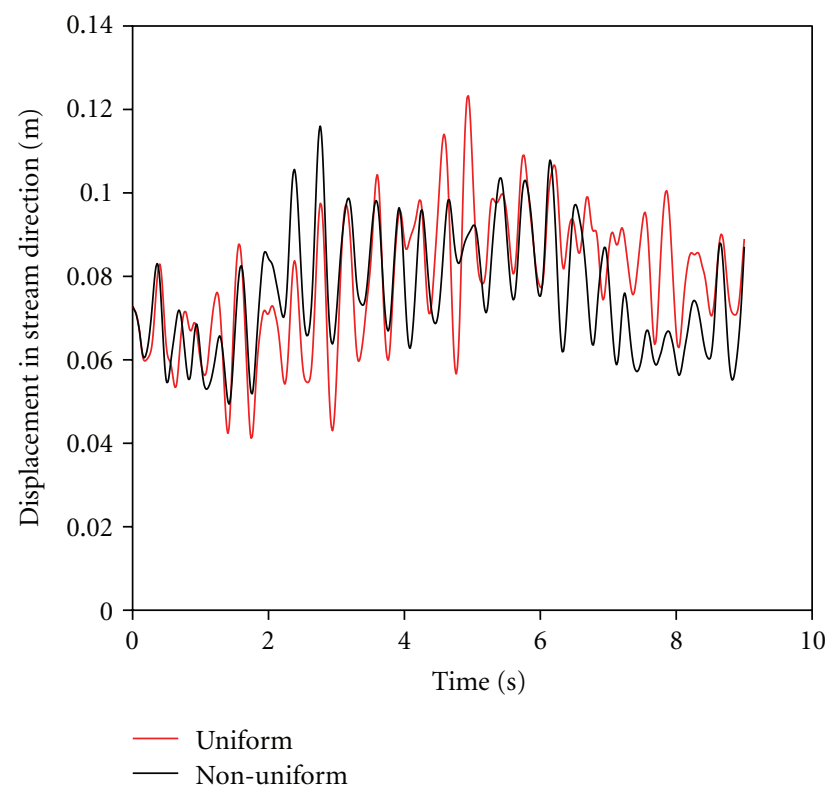

FIgURE 3: Time-history of crest displacement in stream direction.

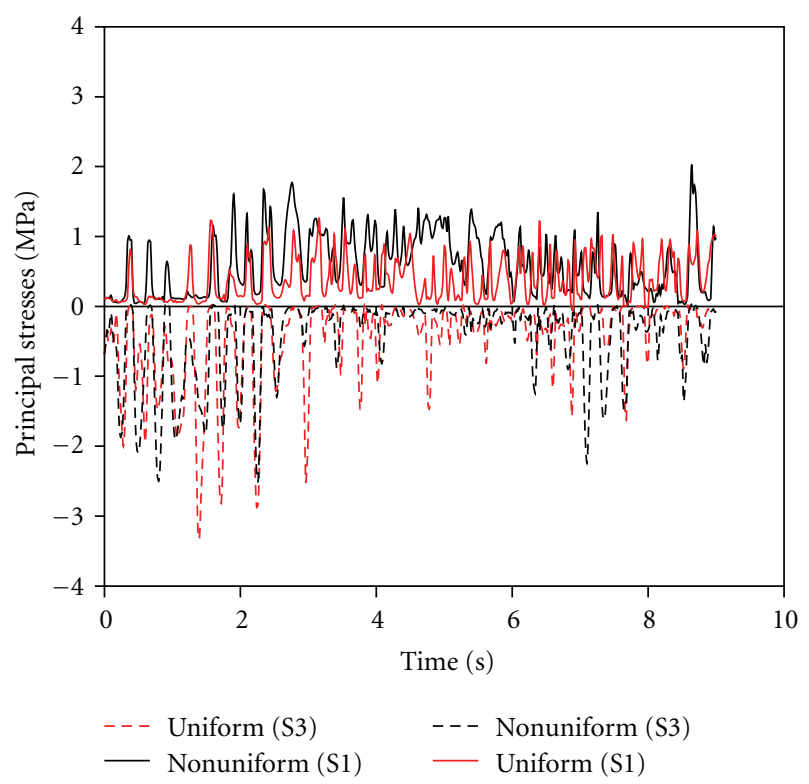

FIgURE 4: Time-history of crest first and third principal stresses.

Figure 4 shows time history of principal stresses at crest point of central cantilever based on uniform and nonuniform analyses. As it is clear using nonuniform excitation leads to higher values in first principal stress (S1) and lower values in third principal stress (S3) for considered point. The general trend of stress variation for both excitations is almost same.

Figures 5 and 6 display nonconcurrent envelopes of principal stresses extracted from uniform and nonuniform analyses on upstream and downstream faces of the dam body for DBL. As can be seen, in nonuniform excitation, maximum $\mathrm{S} 1$ is $9.69 \mathrm{MPa}$ which occurred in the lower part of the dam body at the interface of dam-Pulvino, but in

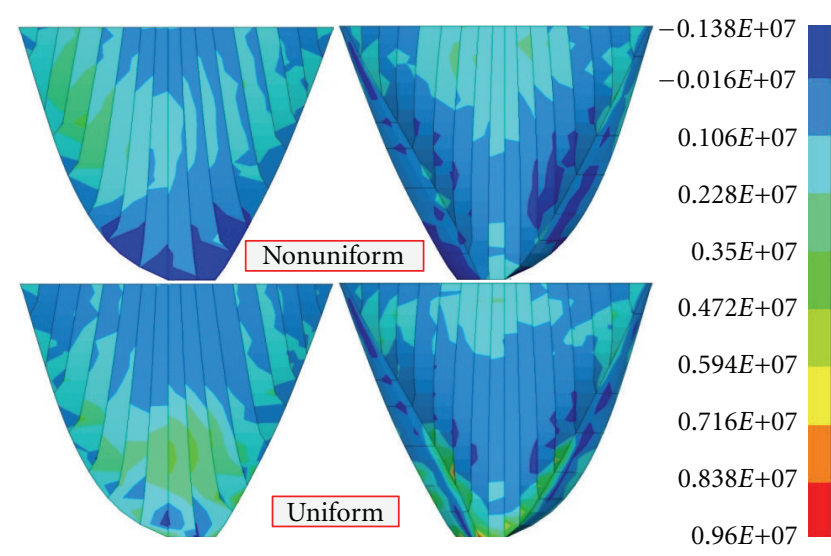

FIGURE 5: Nonconcurrent envelopes of first principal stresses in upstream and downstream faces of dam body $(\mathrm{Pa})$.

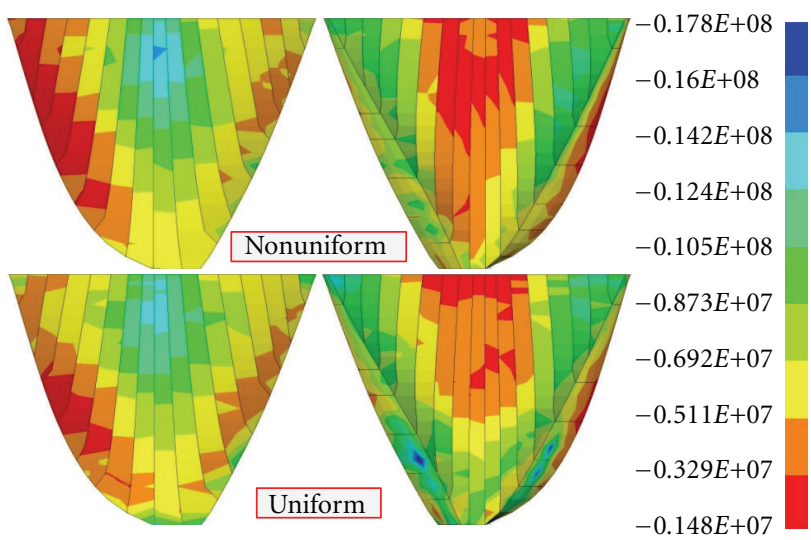

FIGURE 6: Nonconcurrent envelopes of third principal stresses in upstream and downstream faces of dam body $(\mathrm{Pa})$.

uniform excitation this value decreases to $3.85 \mathrm{MPa}$ which occurs at the left part of upstream face. Neglecting over stress points in nonuniform excitation, it can be seen that the area with high stress in upstream face shifted from left parts in uniform excitation to lower parts in nonuniform excitation. In uniform excitation minimum S3 is belonging to central part of upstream face and some areas in damPulvino interface, and its value is $-14.3 \mathrm{MPa}$. In nonuniform excitation the general pattern is similar to uniform, except that minimum S3 is $-17.8 \mathrm{MPa}$ in central part of upstream face in vicinity of crest. According to these figures it is easy to find that difference between uniform and nonuniform inputs for $\mathrm{S} 1$ (that can be interpreted as tensile stress) is intensive than S3 (or compressive stress).

\section{Conclusion}

In this paper, effects of nonuniform excitation due to spatially varying ground motions on nonlinear responses of concrete arch dams are studied. In this study Monte-Carlo simulation approach was used for generating spatially nonuniform ground motion. It was observed that using nonuniform input 
leads to lower values for crest displacement in comparison with uniform excitation. Also it was concluded that differences between uniform and nonuniform excitations in first principal stress are more than third principal stress. Totally nonuniform excitation generates higher tensile and compressive stresses in dam body. The ratio of maximum tensile stress in nonuniform to uniform excitation in present study is 2.51 , and the ratio for minimum compressive stress is 1.24 . It is generally observed that the spatially varying earthquake ground motion model has important effects on the stochastic response of the dam-reservoir-foundation system. Therefore, to be more realistic in calculating the dam response, spatially varying earthquake ground motions should be incorporated in the analysis.

\section{References}

[1] A. Der Kiureghian, "A coherency model for spatially varying ground motions," Earthquake Engineering and Structural Dynamics, vol. 25, no. 1, pp. 99-111, 1996.

[2] R. S. Harichandran, A. Hawwari, and B. N. Sweidan, "Response of long-span bridges to spatially varying ground motion a," Journal of Structural Engineering, vol. 122, no. 5, pp. 476-484, 1996.

[3] S. M. Allam and T. K. Datta, "Seismic response of a cablestayed bridge deck under multi-component non-stationary random ground motion," Earthquake Engineering and Structural Dynamics, vol. 33, no. 3, pp. 375-393, 2004.

[4] Y. H. Zhang, Q. S. Li, J. H. Lin, and F. W. Williams, "Random vibration analysis of long-span structures subjected to spatially varying ground motions," Soil Dynamics and Earthquake Engineering, vol. 29, no. 4, pp. 620-629, 2009.

[5] A. Bayraktar and A. A. Dumano冈lu, "The effect of the asynchronous ground motion on hydrodynamic pressures," Computers and Structures, vol. 68, no. 1-3, pp. 271-282, 1998.

[6] A. Bayraktar, A. A. Dumano®lu, and Y. Calayir, "Asynchronous dynamic analysis of dam-reservoir-foundation systems by the Lagrangian approach," Computers and Structures, vol. 58, no. 5, pp. 925-935, 1996.

[7] O. Maeso, J. J. Aznárez, and J. Domínguez, "Effects of space distribution of excitation on seismic response of arch dams," Journal of Engineering Mechanics, vol. 128, no. 7, pp. 759-768, 2002.

[8] S. W. Alves and J. F. Hall, "Generation of spatially nonuniform ground motion for nonlinear analysis of a concrete arch dam," Earthquake Engineering and Structural Dynamics, vol. 35, no. 11, pp. 1339-1357, 2006.

[9] S. W. Alves and J. F. Hall, "System identification of a concrete arch dam and calibration of its finite element model," Earthquake Engineering and Structural Dynamics, vol. 35, no. 11, pp. 1321-1337, 2006.

[10] P. Cacciola and G. Deodatis, "A method for generating fully non-stationary and spectrum-compatible ground motion vector processes," Soil Dynamics and Earthquake Engineering, vol. 31, no. 3, pp. 351-360, 2011.

[11] G. Deodatis, "Non-stationary stochastic vector processes: seismic ground motion applications," Probabilistic Engineering Mechanics, vol. 11, no. 3, pp. 149-167, 1996.

[12] M. Shinozuka and G. Deodatis, "Stochastic process models for earthquake ground motion," Probabilistic Engineering Mechanics, vol. 3, no. 3, pp. 114-123, 1988.
[13] M. A. Hariri Ardebili and H. Mirzabozorg, "Investigation of endurance time method capability in seismic performance evaluation of concrete Arch Dams," Journal of Dam Engineering, vol. 22, pp. 35-64, 2011.

[14] M. A. Hariri Ardebili and H. Mirzabozorg, "Seismic performance evaluation and analysis of major Arch Dams considering material and joint nonlinearity effects," ISRN Civil Engineering, vol. 2012, Article ID 681350, 10 pages, 2010.

[15] M. A. Hariri Ardebili, M. Mirzabozorg, M. Ghaemian, A. Akhavan, and A. Amini, "Calibration of 3D FE model of DEZ high arch dam in thermal and static conditions using instruments and site observation," in Proceedings of the 6th International Conference on Dam Engineering, pp. 121-135, Lisbon, Portugal, 2011. 

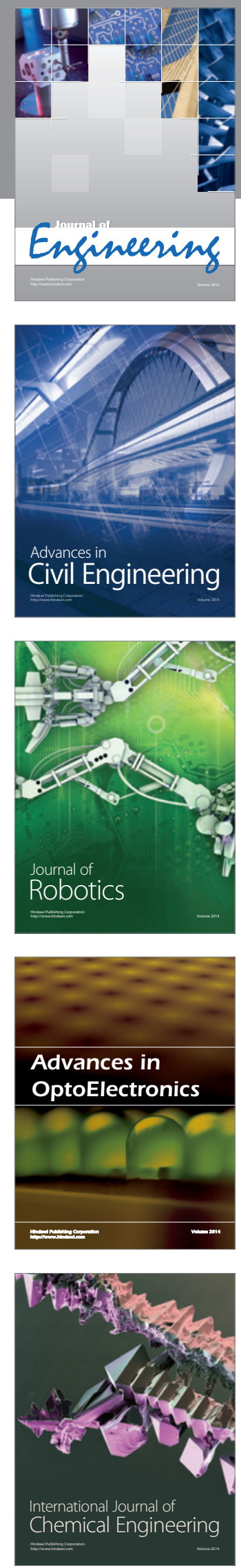

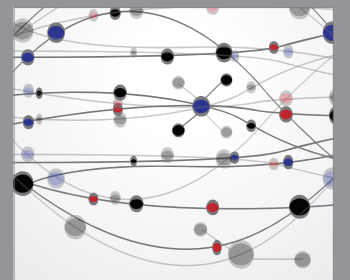

The Scientific World Journal
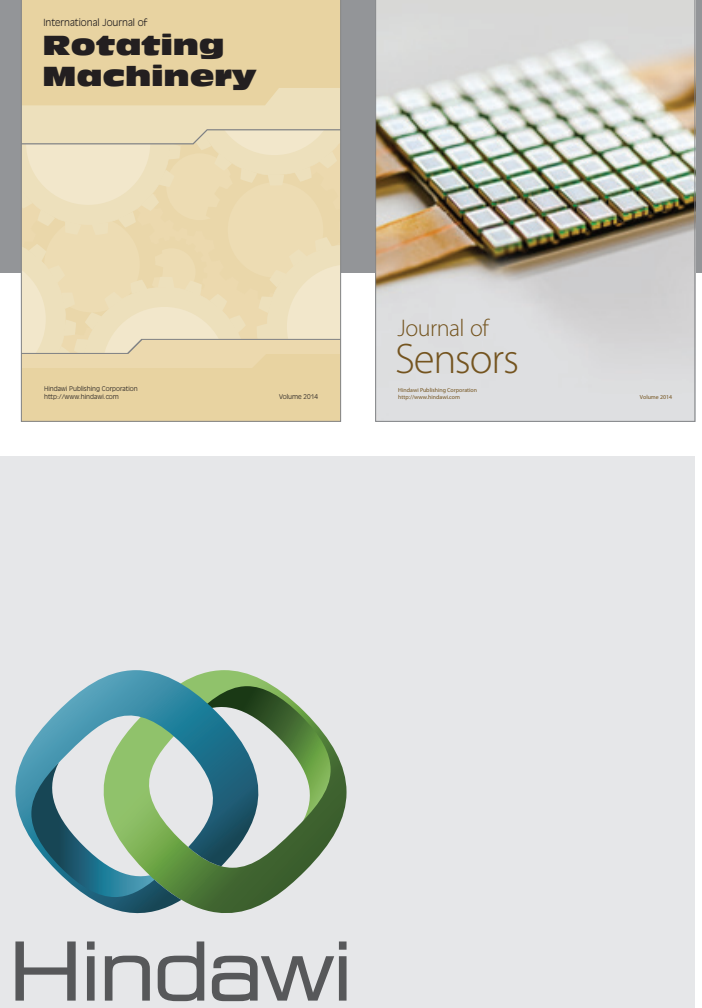

Submit your manuscripts at http://www.hindawi.com
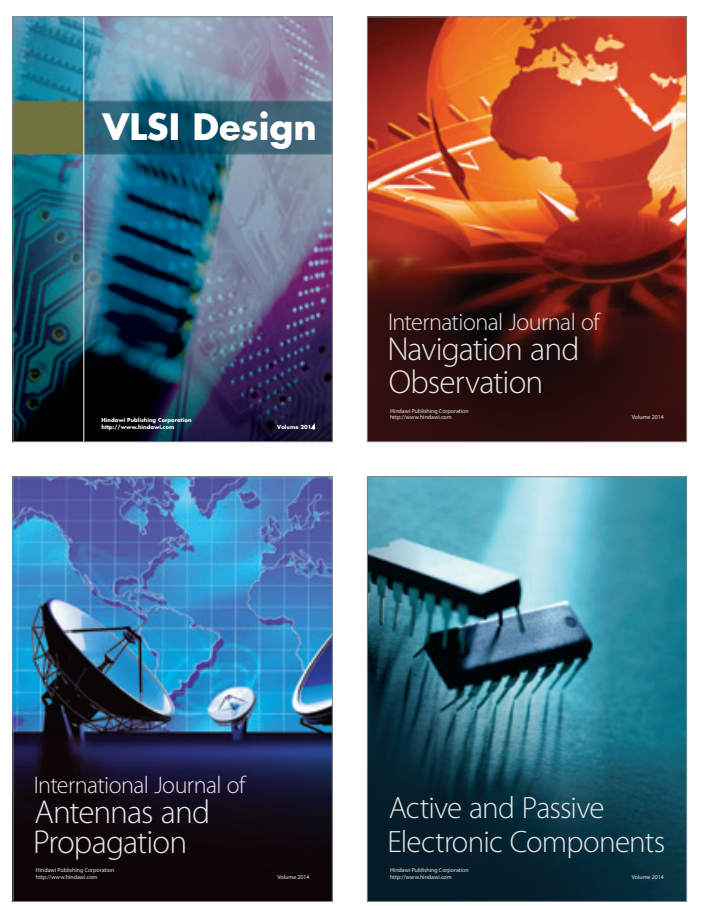
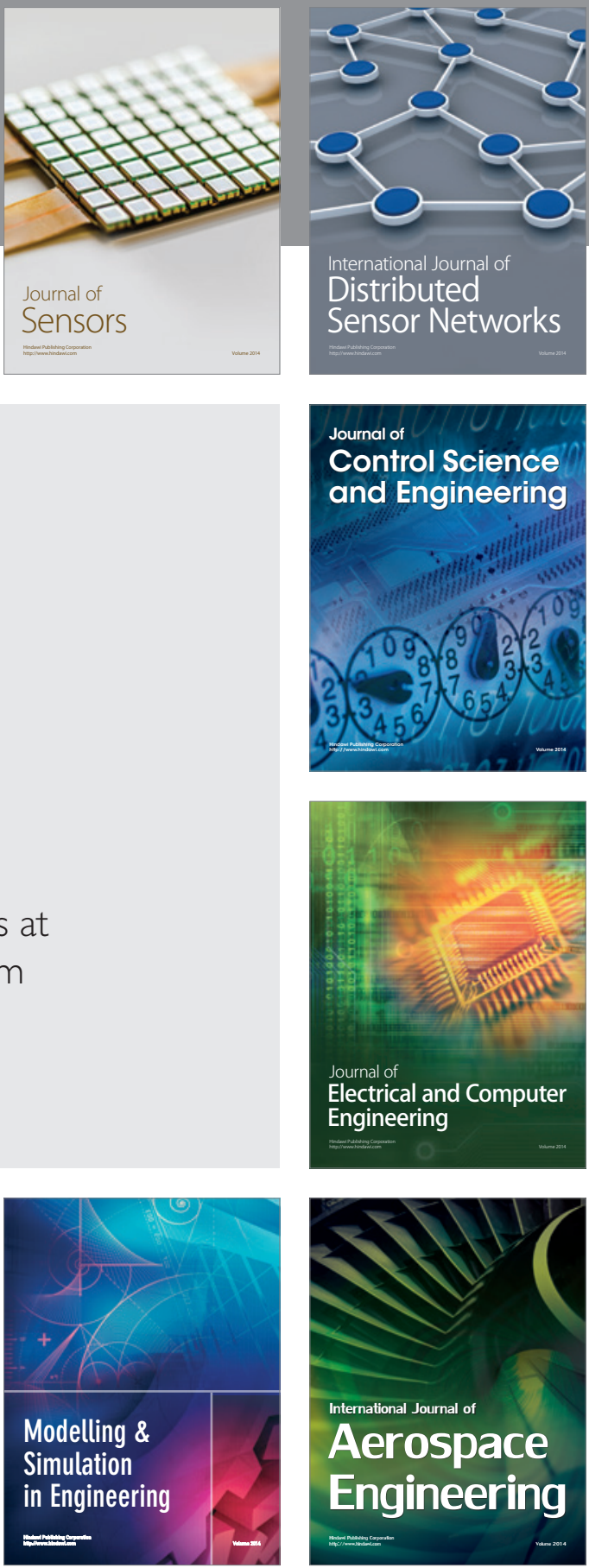

Journal of

Control Science

and Engineering
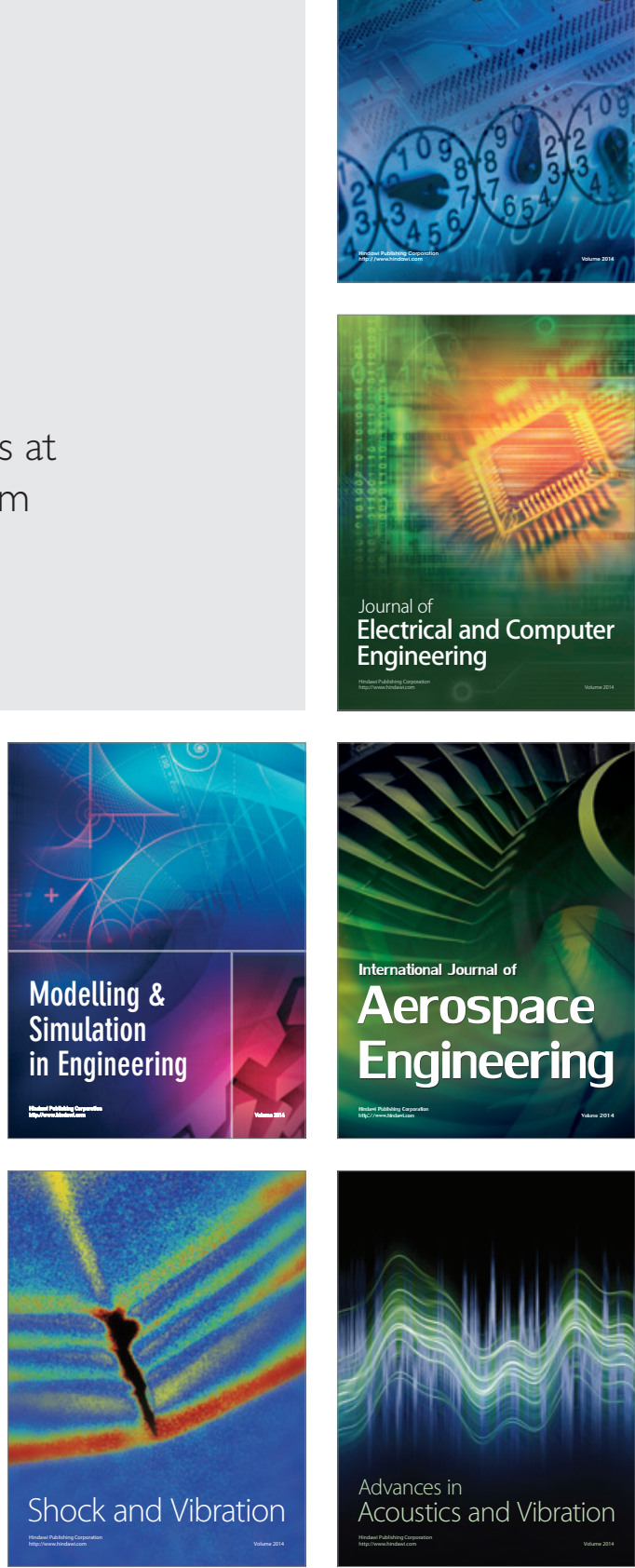